\title{
Theoretical Calculation of Lift Force for General Electric Asymmetric Capacitor Loaded by High Voltage
}

\author{
Yan Zhang1, Xiangyu Cheng2,3*, Pengcheng Huang2, Donghui Jiang'2, Shili Jiang2, \\ Xinxing Qian², Hangwei Ding², Guangli Kuang'2, Wenge Chen² \\ ${ }^{1}$ Anhui Technical College of Water Resources and Hydroelectric Power, Hefei, China \\ ${ }^{2}$ Hefei Institute of Physical Science, Chinese Academy of Science, Hefei, China \\ ${ }^{3}$ No. 38 Research Institute of CETC, Hefei, China \\ Email: ${ }^{\star}$ chanceller@163.com
}

How to cite this paper: Zhang, Y., Cheng, X.Y., Huang, P.C., Jiang, D.H., Jiang, S.L., Qian, X.X., Ding, H.W., Kuang, G.L. and Chen, W.G. (2020) Theoretical Calculation of Lift Force for General Electric Asymmetric Capacitor Loaded by High Voltage. Engineering, 12, 41-46.

https://doi.org/10.4236/eng.2020.121004

Received: December 18, 2019

Accepted: January 13, 2020

Published: January 16, 2020

Copyright $\odot 2020$ by author(s) and Scientific Research Publishing Inc. This work is licensed under the Creative Commons Attribution International License (CC BY 4.0).

http://creativecommons.org/licenses/by/4.0/

\begin{abstract}
Lift force of an asymmetric capacitor in arbitrary shape still cannot be precisely calculated. For an irregular surface of a general asymmetric capacitor of which electric charge is nonuniformly distributed, we dedicate effort to filling the gap of lift force calculation problem. Through theoretical derivation, a general formula result is achieved. By applying theoretical formula combining with experimental method based on similarity principle of dimensional analysis, we can almost figure out all types of lift force of asymmetric capacitor. It is really an exciting method which can universally solve the hard thrust force calculation problem completely.
\end{abstract}

\section{Keywords}

High-Voltage, Asymmetric Capacitor, Lift Force, Calculation, General

\section{Introduction}

A formula $f=\frac{q^{2}}{\varepsilon}\left(\frac{1}{S_{1}}-\frac{1}{S_{2}}\right)$ was performed for a kind of ideal asymmetric capacitor [1] in former paper. It looks simple and brief. But there are still a series of problems that need us to solve like the quantity of electric charges and what's the surface distribution. The formula is suitable for an ideal asymmetric capacitor and its surface electric charges are uniformly distributed. But for an irregular surface of a general asymmetric capacitor of which electric charges are nonuniformly distributed, how can we figure out the lifter force is the main problem we 
will further analyze. That is to say, we will make the lifter force calculation formula of asymmetric capacitor generalizations.

We derived the lift force formula of asymmetric capacitor [2] in ideal case. But in normal conditions, asymmetric capacitor isn't in an ideal regular shape (refer Figure 1). So we must expend the equation into general case suitable for practical engineering. In the next content below, we will show the lift force formula of asymmetric capacitor in general firstly, and then, the derivation process is given out.

For a normal asymmetric capacitor, after loading with a DC high-voltage on its two plates, the lift force produced on the capacitor is

$$
f=\frac{1}{\varepsilon q} \int_{0}^{1 t} \int_{0}^{2 t} \sigma_{1}\left(S_{1}\right) \sigma_{2}\left(S_{2}\right) \cdot\left[\sigma_{1}\left(S_{1}\right)-\sigma_{2}\left(S_{2}\right)\right] \cdot \mathrm{d} S_{2} \mathrm{~d} S_{1}
$$

where $f$ is lift force of asymmetric capacitor, $q$ is carried electric charge of the plates, $\varepsilon=\varepsilon_{r} \varepsilon_{0}$ is permittivity, $S_{1}$ is area of small plate, $S_{2}$ is area of large plate, $\sigma(S)$ is charge density distribution function relevant to geometric shape and location.

\section{Derivation Process}

The lift force calculation formula is shown in Equation (1). This is suitable for a general asymmetric which surface charge is normally uneven distributed on most of actual situations [3] [4] [5] [6] [7]. The detail derivation process is expanded as follows. Before concrete derivation, we will list some preconditions and steps.

The derivation starting point is established on these assumption conditions:

1) Electric field line is always closed. All of the Electric field lines emit from positive electrode patches, return to negative electrode patches, but can never be blocked by others neutral objects. Total charge carried on the positive electrode $q=\int \mathrm{d} q_{1}=\int \varepsilon E_{1} \cdot \mathrm{d} S_{1}$ is ever equal to that on the negative electrode $q=\int \mathrm{d} q_{2}=\int \varepsilon E_{2} \cdot \mathrm{d} S_{2}$.

2) For a different element area on a negative electrode, the more charge it carries, the more electric field strength it owns. In other words, the electric field strength is distributed in proportion to the negative charge it carried.

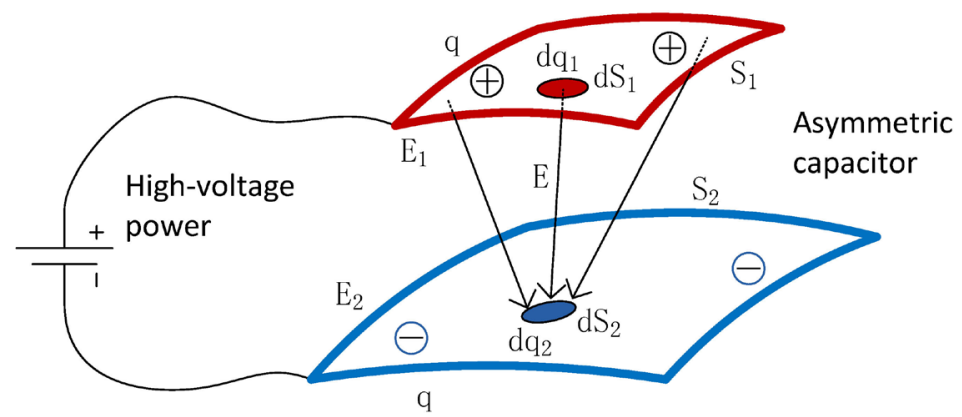

Figure 1. An asymmetric capacitor of nonuniform distribution of electric charge in general case. 
3) Similarly, for a different element area on a positive electrode, the more charge it carries, the more electric field strength it emits to send out. In other words, the electric field strength is distributed in proportion to the positive charge it carried.

Initial conditions:

There are 2 capacitor plates: (small) plate 1 and (large) plate 2 . They are separately electrical charged with $\pm q$. We take different operating patch area: $\mathrm{d} S_{1}$ on plate 1 and $\mathrm{d} S_{2}$ on plate 2 . And meanwhile make sure that areas of the two different operating patches are the same. That is to say, it satisfies $\mathrm{d} S_{1}=\mathrm{d} S_{2}=\mathrm{d} S$.

Based on these conditions, we have following corollaries.

For plate 1, there are

$$
\begin{gathered}
\mathrm{d} q_{1}=\sigma_{1}\left(S_{1}\right) \cdot \mathrm{d} S_{1}, \quad S_{1} \in\left[0, S_{1 t}\right] \\
E_{d s 1}=\frac{\sigma_{1}\left(S_{1}\right)}{\varepsilon}=\frac{\mathrm{d} q_{1}}{\varepsilon \cdot \mathrm{d} S_{1}} \\
q_{10}=0 \\
q_{1 t}=\int_{0}^{S_{1 t}} \mathrm{~d} q_{1}=\int_{0}^{S_{1 t}} \sigma_{1}\left(S_{1}\right) \cdot \mathrm{d} S_{1}=q
\end{gathered}
$$

For plate 2, there are similarly

$$
\begin{gathered}
\mathrm{d} q_{2}=\sigma_{2}\left(S_{2}\right) \cdot \mathrm{d} S_{2}, \quad S_{2} \in\left[0, S_{2 t}\right] \\
E_{d s 2}=\frac{\sigma_{2}\left(S_{2}\right)}{\varepsilon}=\frac{\mathrm{d} q_{2}}{\varepsilon \cdot \mathrm{d} S_{2}} \\
q_{20}=0 \\
q_{2 t}=\int_{0}^{S_{2 t}} \mathrm{~d} q_{2}=\int_{0}^{S_{2 t}} \sigma_{2}\left(S_{2}\right) \cdot \mathrm{d} S_{2}=q
\end{gathered}
$$

So, the electric field strength nearby plate 2 is $E_{d S 2}=\frac{\mathrm{d} q_{2}}{\varepsilon \cdot \mathrm{d} S_{2}}$. From the first and the third assumption conditions, we known that: the total electric field lines $E_{d S 2}$ emitted from plate 2, not only distribute to the area differential element $\mathrm{d} s_{1}$ on plate 1 (which is charged with electric quantity $\mathrm{d} q_{1}$ ), but also distribute to the rest of area (the total area corresponding to $\mathrm{d} s_{2}$ ) on plate 1 (which is charged with total electric quantity $q$ ). Hence the area differential element $\mathrm{d} s_{1}$ actually achieves a electric field strength $E_{d S 1 h}=E_{d S 2} \frac{\mathrm{d} q_{1}}{q}$ which is derived from $\mathrm{d} s_{2}$.

The assumption of equal electric density causes the remaining charge produced on one differential element of plate 1 which electric density is actually higher than plate 2. The remaining charge $\Delta q=\mathrm{d} q_{1}-\mathrm{d} q_{2}$ on one differential element of plate $1 \mathrm{~d} s_{1}$ which satisfies $\mathrm{d} S_{1}=\mathrm{d} S_{2}=\mathrm{d} S$.

So we can get the force produced by remaining charge $\Delta q$ in the assumption electric field $E_{d S 1 h}$

$$
\mathrm{d} f=\Delta q \cdot E_{d S 1 h}=\Delta q \cdot E_{d S 2} \frac{\mathrm{d} q_{1}}{q}=\left(\mathrm{d} q_{1}-\mathrm{d} q_{2}\right) \cdot \frac{\mathrm{d} q_{2}}{\varepsilon \cdot \mathrm{d} S_{2}} \cdot \frac{\mathrm{d} q_{1}}{q}
$$


Integrating Equation (6), we have

$$
\begin{aligned}
f & =\int_{0}^{1 t} \int_{0}^{2 t} \mathrm{~d} f=\int_{0}^{1 t} \int_{0}^{2 t}\left(\mathrm{~d} q_{1}-\mathrm{d} q_{2}\right) \cdot \frac{\mathrm{d} q_{2}}{\varepsilon \cdot \mathrm{d} S_{2}} \cdot \frac{\mathrm{d} q_{1}}{q} \\
& =\int_{0}^{1 t} \int_{0}^{2 t}\left[\sigma_{1}\left(S_{1}\right) \cdot \mathrm{d} S_{1}-\sigma_{2}\left(S_{2}\right) \cdot \mathrm{d} S_{2}\right] \cdot \frac{\sigma_{2}\left(S_{2}\right) \cdot \mathrm{d} S_{2}}{\varepsilon \cdot \mathrm{d} S_{2}} \cdot \frac{\sigma_{1}\left(S_{1}\right) \cdot \mathrm{d} S_{1}}{q}
\end{aligned}
$$

Combining the initial condition $\mathrm{d} S_{1}=\mathrm{d} S_{2}=\mathrm{d} S$, we can further simplify above equation

$$
\begin{aligned}
f & =\int_{0}^{1 t} \int_{0}^{2 t}\left[\sigma_{1}\left(S_{1}\right) \cdot \mathrm{d} S_{2}-\sigma_{2}\left(S_{2}\right) \cdot \mathrm{d} S_{2}\right] \cdot \frac{\sigma_{2}\left(S_{2}\right) \cdot \mathrm{d} S_{2}}{\varepsilon \cdot \mathrm{d} S_{2}} \cdot \frac{\sigma_{1}\left(S_{1}\right) \cdot \mathrm{d} S_{1}}{q} \\
& =\int_{0}^{1 t} \int_{0}^{2 t} \frac{\sigma_{1}\left(S_{1}\right) \cdot \sigma_{2}\left(S_{2}\right)}{\varepsilon q}\left[\sigma_{1}\left(S_{1}\right)-\sigma_{2}\left(S_{2}\right)\right] \cdot \mathrm{d} S_{2} \mathrm{~d} S_{1} \\
& =\frac{1}{\varepsilon q} \int_{0}^{1 t} \int_{0}^{2 t} \sigma_{1}\left(S_{1}\right) \sigma_{2}\left(S_{2}\right) \cdot\left[\sigma_{1}\left(S_{1}\right)-\sigma_{2}\left(S_{2}\right)\right] \cdot \mathrm{d} S_{2} \mathrm{~d} S_{1}
\end{aligned}
$$

So we get the lift force formula in general form.

\section{Similarity Principle}

Equation (8) of calculating lift force is based on knowing electric density distribution function $\sigma(S)$. But in most situations, the electric density distribution is normally unknown. In this case, we should use theoretical combining with experimental method based on similarity principle of dimensional analysis to figure out the final force produced between two plates. The detail steps should be as follows.

1) Establishing a smaller model in scale $1: a(a>1)$ that is similar to the original asymmetric capacitor.

2) Applying a voltage to the miniature model of which the value is $1 / a$ of the original.

3) Electrifying the asymmetric capacitor, and actually measuring the lift force produced by the miniature model which is $f_{2}$.

4) Achieving the lift force of original model which is equal to square value of the small similar one, using magnifying method $f_{1}=a^{2} f_{2}$.

Before calculating the final lift force of the right side lifter (refer Figure 2), we may measure lift force of a small scale model similar to it firstly, and then achieve the lift force of original model by scale magnifying method. If we measured the lift force is $0.2 \mathrm{~N}$ on the left side lifter, the lift force of original right side lifter (10 times larger than the left side one) is $f_{1}=a^{2} f_{2}=10^{2} \times 0.2=20 \mathrm{~N}$.

\section{Affiliated Verification}

A brief verification is performed by simplifying Equation (8) from general to special case. If the both plates of an ideal asymmetric capacitor are distributed with uniform charge, there are following relations.

Firstly, we can take a part area from plate $1 S_{1 p}$ whose electric quantity is 


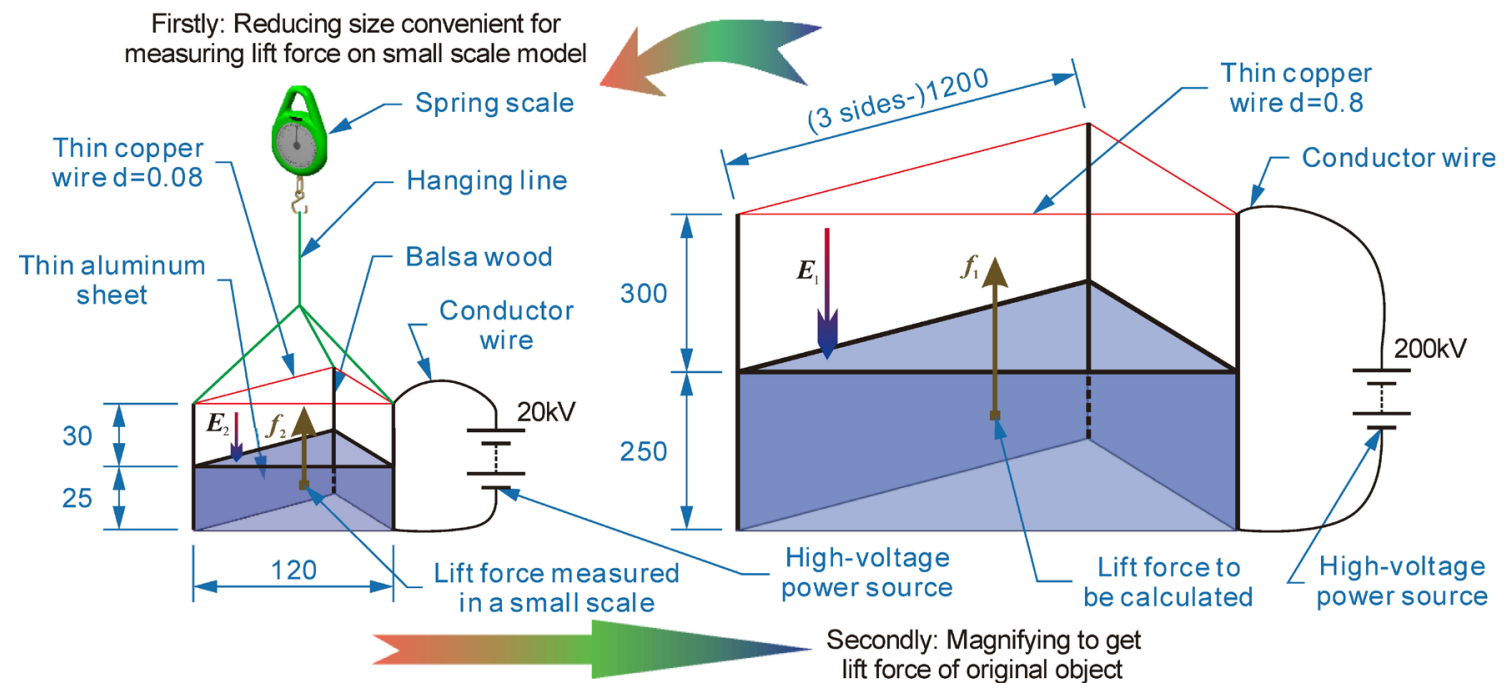

Figure 2. Scale magnifying method to calculate lift force of arbitrarily shaped asymmetric capacitor (or called lifter).

$$
q_{1 p}=\int_{0}^{S_{1 p}} \sigma_{1}\left(S_{1}\right) \cdot \mathrm{d} S_{1}=\frac{S_{1 p}}{S_{1 t}} \cdot q
$$

So we get the distribution function of electric charge density that is

$$
\sigma_{1}\left(S_{1}\right)=\frac{q_{1 p}}{S_{1 p}}=\frac{q}{S_{1 t}}
$$

Secondly, we can take a part area from plate $2 S_{2 p}$ whose electric quantity is

$$
q_{2 p}=\int_{0}^{S_{2 p}} \sigma_{2}\left(S_{2}\right) \cdot \mathrm{d} S_{2}=\frac{S_{2 p}}{S_{2 t}} \cdot q
$$

So we get the distribution function of electric charge density that is

$$
\sigma_{2}\left(S_{2}\right)=\frac{q_{2 p}}{S_{2 p}}=\frac{q}{S_{2 t}}
$$

Lastly, plugging the Equations (10) and (12) of special case of uniformly distribution of electric charge into Equation (8) of general case of nonuniform distribution of electric charge, we have

$$
\begin{aligned}
f & =\frac{1}{\varepsilon q} \int_{0}^{1 t} \int_{0}^{2 t} \sigma_{1}\left(S_{1}\right) \sigma_{2}\left(S_{2}\right) \cdot\left[\sigma_{1}\left(S_{1}\right)-\sigma_{2}\left(S_{2}\right)\right] \cdot \mathrm{d} S_{2} \mathrm{~d} S_{1} \\
& =\frac{1}{\varepsilon q} \int_{0}^{1 t} \int_{0}^{2 t} \frac{q}{S_{1 t}} \frac{q}{S_{2 t}} \cdot\left(\frac{q}{S_{1 t}}-\frac{q}{S_{2 t}}\right) \cdot \mathrm{d} S_{2} \mathrm{~d} S_{1} \\
& =\frac{1}{\varepsilon q} \cdot \frac{q}{S_{1 t}} \frac{q}{S_{2 t}} \cdot\left(\frac{q}{S_{1 t}}-\frac{q}{S_{2 t}}\right) \int_{0}^{1 t} \int_{0}^{2 t} \mathrm{~d} S_{2} \mathrm{~d} S_{1} \\
& =\frac{q^{2}}{\varepsilon} \cdot \frac{1}{S_{1 t}} \frac{1}{S_{2 t}} \cdot\left(\frac{1}{S_{1 t}}-\frac{1}{S_{2 t}}\right) \cdot S_{2 t} S_{1 t} \\
& =\frac{q^{2}}{\varepsilon} \cdot\left(\frac{1}{S_{1 t}}-\frac{1}{S_{2 t}}\right)
\end{aligned}
$$

Therefore, we can finally find that the lastly corollary is as the same as the 
former formula for calculating lift force of electric charge at ideal uniformly distribution in special case.

\section{Conclusion}

From above derivation, we get the general calculation formula of lift force of asymmetric capacitor. And by further simplification, we finally find the formula in ideal case is the same as previous deduction. The general calculation method can help us to get the lift force of asymmetric capacitor precisely in any shape. It is really an exciting method which can universally solve the hard thrust calculation problem completely.

\section{Acknowledgements}

The authors gratefully acknowledge the support of the Thirteenth Five-Year Plan of Hefei Institute of Physical Science of Chinese Academy of Science (Grant No. Y86CT21051 "Electric and Magnetic Propulsion System"), the Research Activity Funding of Postdoctoral Fellow of Anhui Province (Grant No. 2018B250 "High-energy Ions Accelerated Thruster"), the Natural Science Research Project of Anhui Education Department (Grant No. KJ2018A0725 "the Uniformity Optimization and Software Development for MRI Magnets"), and a portion of this work was supported by the High Magnetic Field Laboratory of Anhui Province.

\section{Conflicts of Interest}

The authors declare no conflicts of interest regarding the publication of this paper.

\section{References}

[1] Zhang, J.T., et al. (2011) A High-Performance Asymmetric Supercapacitor Fabricated with Graphene-Based Electrodes. Energy \& Environmental Science, 4, 4009-4015. https://doi.org/10.1039/c1ee01354h

[2] Lezana, P., Aguilera, R. and Quevedo, D.E. (2009) Model Predictive Control of an Asymmetric Flying Capacitor Converter. IEEE Transactions on Industrial Electronics, 56, 1839-1846. https://doi.org/10.1109/TIE.2008.2007545

[3] Bahder, T. and Fazi, Ch. (2003) Force on an Asymmetrical Capacitor. Army Report ARL-TR-3005. Army Res. Lab., Adelphi.

[4] Brown, T.T. (1928) A Method of and an Apparatus or Machine for Producing Force or Motion. UK Patent, No. 300311.

[5] Primas, J., Malík, M., Jašíková, D. and Kopecký, V. (2010) Force on High Voltage Capacitor with Asymmetrical Electrodes. Processing of the WASET 2010 Conference, Amsterdam, 335-339.

[6] Canning, F.X., Melcher, C. and Winet, E. (2004) Asymmetrical Capacitors for Propulsion. Institute for Scientific Research, Inc., Fairmont, West Virginia.

[7] Long, K. (2012) The Role of Speculative Science in Driving Technology. In: Deep Space Propulsion, Springer, New York, NY, 287-304.

https://doi.org/10.1007/978-1-4614-0607-5_16 